Classification

Physics Abstracts

$78.70 \mathrm{D}-78.90$

\title{
Strategies for determination of inter-atomic distances from extended energy loss fine structure
}

\author{
Mohammad A. Tafreshi, Stefan Csillag, Zou Wei Yuan and Christian Bohm \\ Institute of Physics, University of Stokholm, Vanadisvägen 9, S-113 46 Stockholm, Sweden
}

(Receveid June 28, 1991; accepted October 02, 1991)

\begin{abstract}
In Extended Energy Loss Fine Structure spectroscopy (EXELFS), modulations occuring on the high energy slope of the ionization edges in electron energy loss spectra are investigated. These modulations supply important structural and chemical information about the local atomic environment of the absorbing atoms. The accuracy and the reproducibility of the results, however, are greatly influenced by the procedures used in the data analysis. In this paper a detailed study of the analyzing method for determination of the interatomic distances is presented. A reliable FFT based deconvolution procedure for extending the method to thick samples is also described.
\end{abstract}

\section{Introduction.}

The modulations occuring on the high energy side of the ionization edge in electron energy loss spectrometry, the EXtended Energy Loss Fine Structure (EXELFS), are due to the interference between outgoing and backscattered parts of the ejected core electron wave. The latter part is caused by the scatter of the electron wave against the neighboring atoms. The interference can be constructive or destructive depending on the energy (or rather the wave length) of the excited electron and the distance to the neighboring atoms. Constructive interference will result in an increasing ionization probability while the converse is true for destructive interference [1].

Approximating the ejected electron wave function at the backscattering atom by a plane wave while assuming single scattering formalism with small scattering angle, the interference amplitude can be described [2], by:

$$
X(k)=\sum_{j} \frac{N_{j}}{r_{j}^{2}} \cdot\left(\frac{f_{j}(k)}{k} \cdot \mathrm{e}^{-2 \cdot \sigma_{j}^{2} \cdot k^{2}} \cdot \mathrm{e}^{-2 \cdot r_{j} / \lambda_{\mathrm{i}}}\right) \cdot \sin \left(2 k r_{j}+\phi_{j}(k)\right)
$$

where $N_{j}$ is the number of atoms in shell $j, r_{j}$ is the radius, $\phi_{j}(k)$ is the phase difference between the outgoing and reflected waves. $f_{j}(k)$ is the backscattering amplitude which depends on the type of atoms and which for light atoms can be approximated by $1 / k^{2}$ [3]. $\lambda_{\mathrm{i}}$ is the mean free path for inelastic scattering of the ejected electron i.e. a function of its kinetic energy, and $\sigma_{j}$ is a parameter describing the thermal vibration and static disorder. The expression provides means 
for deriving information about the local environment of the excited atom from an electron energyloss spectrum. In addition to the interatomic distances, $r_{j}$, the disorder parameter and the number of atoms at the distances $r_{j}$, can be extracted.

Many of the procedures used in EXELFS analysis are similar to the closely related Extended $\mathrm{X}$-ray Absorption Fine Structure (EXAFS) data analysis. There are, however, a number of significant differences. These originate from the amount of plural scattering, the momentum transfer dependence, the short data range, and from different background effects. Some of these effects can be reduced by a careful selection of the experimental conditions, others have to be recognized and properly dealt with during the data analysis.

The analysis consists of different steps, which will be closely examined in the following. Two spectra from a graphite sample, one from a thin region and the other from a thicker region are used as examples.

\section{Method and analysis.}

PRE-EDGE BACKGROUND SUBTRACTION. - This step in the analysis is primarily concerned with removing other absorption effects than those related to the edge under study. Inner-shell ionization edges are mostly superimposed upon monotonically decreasing backgrounds which originate from the excitation of atomic electrons of lower binding energy. After approximating this background with a smooth decreasing function it may be removed from the spectrum. This is done by fitting a smooth function to the data below the edge, extrapolating it into the core-loss region and subtracting it from the measured data [4].

Several attempts to fit polynomial or exponential functions to the pre-edge region have been reported. However, none of these methods have proved to be consistently reliable since there is no guarantee that the extension of a smooth function based only on a few points within the pre-edge region, should accurately describe the background in the core-loss and particularly in the higher energy-loss region. A small error in the determination of this smooth function could drastically change the shape of the background at the high energy end, which in some cases can cause an unphysical intersect between the extrapolated part of the background and the spectrum. Moreover it is desirable that the background assymptotically approaches the high energy side of the spectrum in order to minimize the truncation effects after deconvolution.

The above requirements implies that when modeling the pre-edge background, considerations should be taken both to the pre-edge and to the high energly-loss region of the spectrum. The following function can be used:

$$
F(i)=A+\frac{B}{i+C}
$$

where $A$ corresponds to an energy independent part of the background, $B$ and $C$ are free parameters and $i$ is the channel number. After $A$ has been chosen as the estimated constant background level, $B$ and $C$ are determined by least square fitting of $F$ to the pre-edge region. If no direct measurements of the constant background are available, a reasonable estimate can be made by choosing a fraction of the value corresponding to the largest energy loss as the constant background.

As will be shown below, a further background removal is necessary to isolate the oscillatory part of the EXELFS. This second background elimination also has the effect that it reduces remaining pre-edge contributions.

Figures $1 \mathrm{a}$ and $1 \mathrm{~b}$ show core-loss spectra from a thin and a thicker region of a graphite sample. Their corresponding low-loss spectra are shown in figures 1c and 1d, respectively. Figure 1e is the same spectrum as in figure 1a but this time overlaid with a pre-edge background obtained by 
fitting equation (2) to pre-edge data and the last point in the core-loss spectrum. Figure 1f shows the spectrum after this background has been subtracted.

CALIBRATION OF THE ENERGY-LOSS AXIS. - By comparing an independently determined plasmon or inner-shell excitation energy from a standard sample, with the channel distance between the corresponding peaks in the energy loss spectrum (the zero-loss peak and the peak with energy-loss due to plasmon or inner-shell excitation), the $\mathrm{eV}$ per channel value can be estimated and used to calibrate the energy-loss axis.

An inaccurate calibration of the energy-axis introduces a constant relative error in $k$ and in the interatomic distances [5], which for small deviations in $E_{\text {ref }}$, should be:

$$
\frac{\Delta r}{r}=-\frac{\Delta E_{\mathrm{ref}}}{2 \cdot E_{\mathrm{ref}}}
$$

In the analysis of the samples presented in this work, the standard experimental value for the first plasmon excitation energy of graphite $(27 \mathrm{eV})$ was used.

REMOVAL OF THE PLURAL SCATTERING FROM INNER-SHELL EDGES. - Plural scattering occurs when an electron suffers one or more inelastic excitations (outer-shell excitation) in combination with the core-loss ionization. Since the probability of these excitations increase with increasing sample thickness, the thickness of the examined sample region will influence the observed shape of the modulations. It will also decrease the signal to background ratio, which in turn degrades the statistics.

Thus the contribution from the plural scattering may have to be removed before the near-edge or the extended fine structure can be interpreted, particularly in the case of thick samples. It should also be noted that, since the mean free path for the core-loss excitations usually is small compared to the sample thicknesses, the probability of more than one core ionization can be neglected.

A Fourier transform based deconvolution technique will be used [6], dividing the Fourier transform of the core-loss region by the Fourier transform of the low-loss region. The latter contains the zero-loss peak and energy losses up to typically $100 \mathrm{eV}$.

In addition to removing the plural scattering contribution, the deconvolution also removes the influence of the system resolution function. The resolution is thus improved but at the expense of greatly amplifying the noise. It is therefore necessary to apply a filter function to smooth the deconvoluted data before continuing analysis. An adequate function for this purpose is a narrow Gaussian [5]. Choosing the width is a trade off between too much noise if the width is too small and deteriorated resolution if the width is too large. In practice, the deconvolution procedure often has to be repeated with different Gaussian functions in order to find the optimum width. However, the width of the zero-loss peak provides a good starting value.

Since the deconvolution process is very sensitive to the defects and artifacts in the data spectrum, a successful removal of plural scattering effects by deconvolution requires accurate spectra.

Figure $2 \mathrm{~b}$ show the energy-loss spectrum of the thick sample i.e. figure $1 \mathrm{~b}$, after pre-edge background subtraction, with and without deconvolution. The Gaussian function used (Fig. 2a), has a width of 5.7 channels.

IDENTIFICATION OF THE THRESHOLD-ENERGY POSITION. - The threshold-energy position should be identified as the origin of the energy axis. Hence selection of an appropriate threshold-energy position has been recognized as an important but difficult problem, which must be confronted when analyzing EXELFS spectrum [7]. 


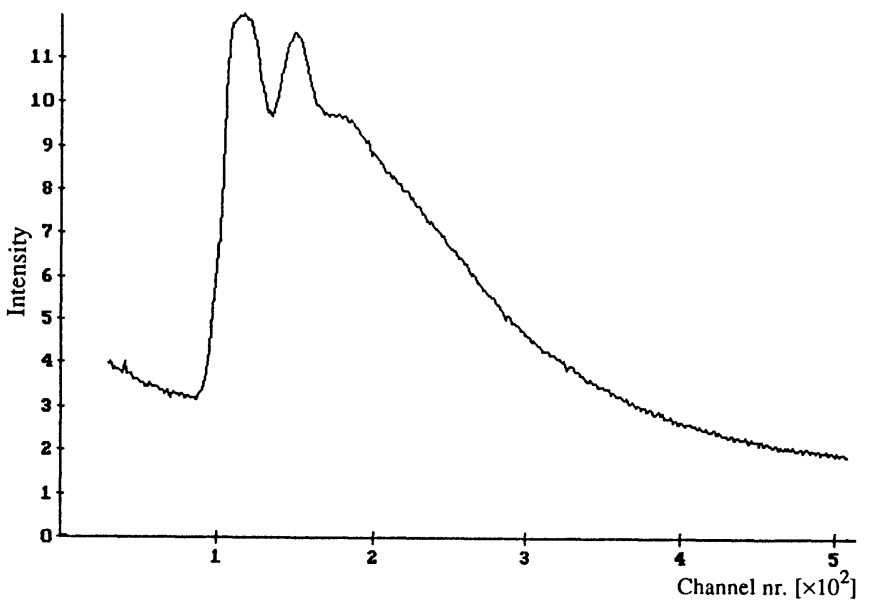

a)

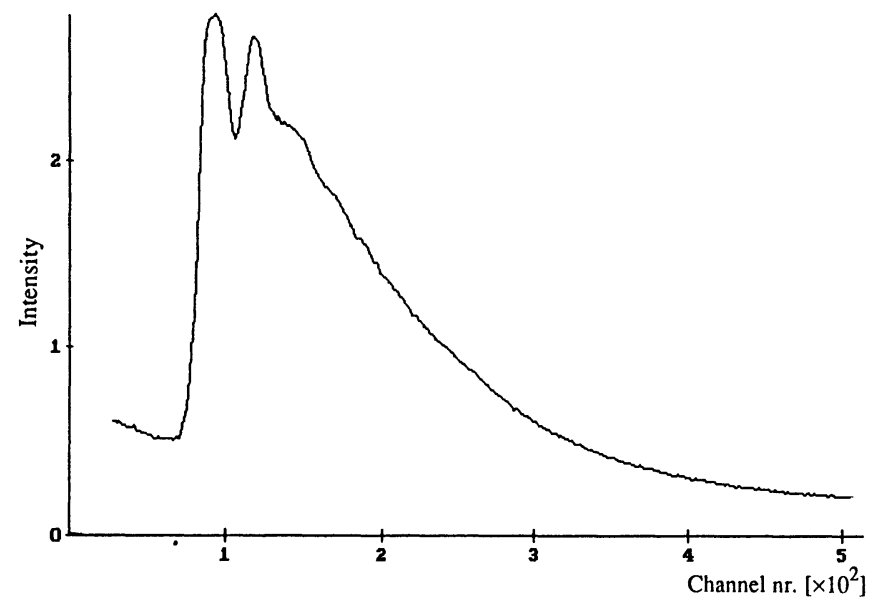

b)

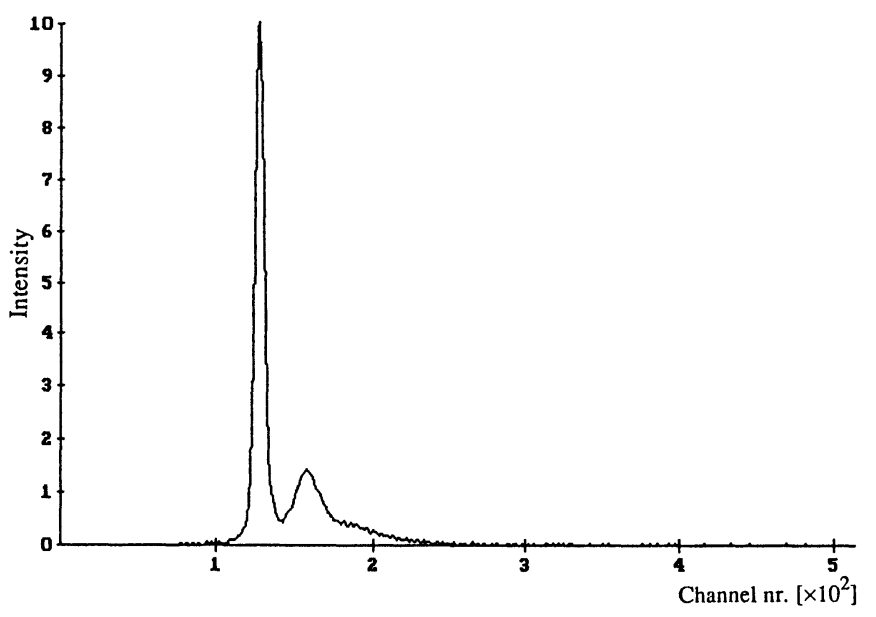

c)

Fig. 1. - (a) and (b) are the energy-loss spectra of a thin and a thicker region of a graphite specimen. (c) and (d) are the corresponding low-loss spectra. (e) is identical with (a) but with a pre-edge background inserted. (f) is the same spectrum after background subtraction. 


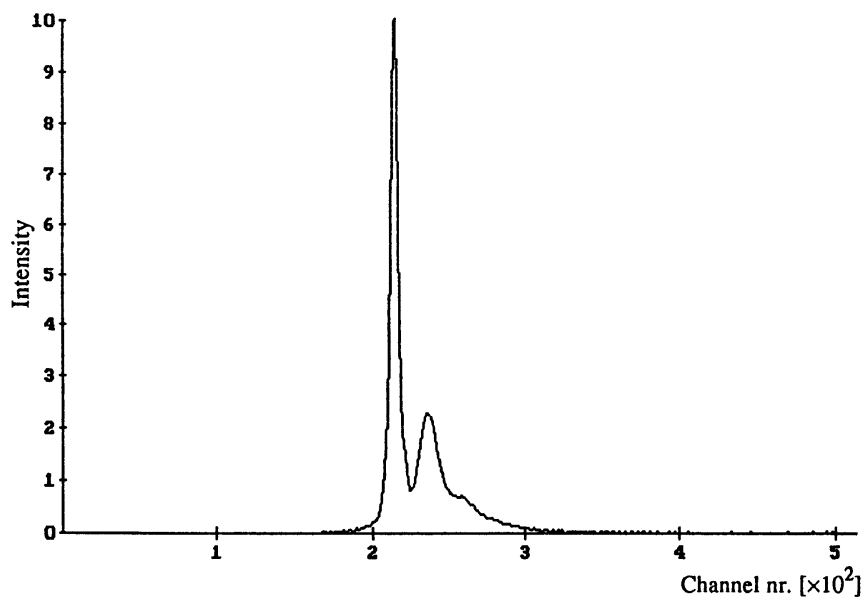

d)

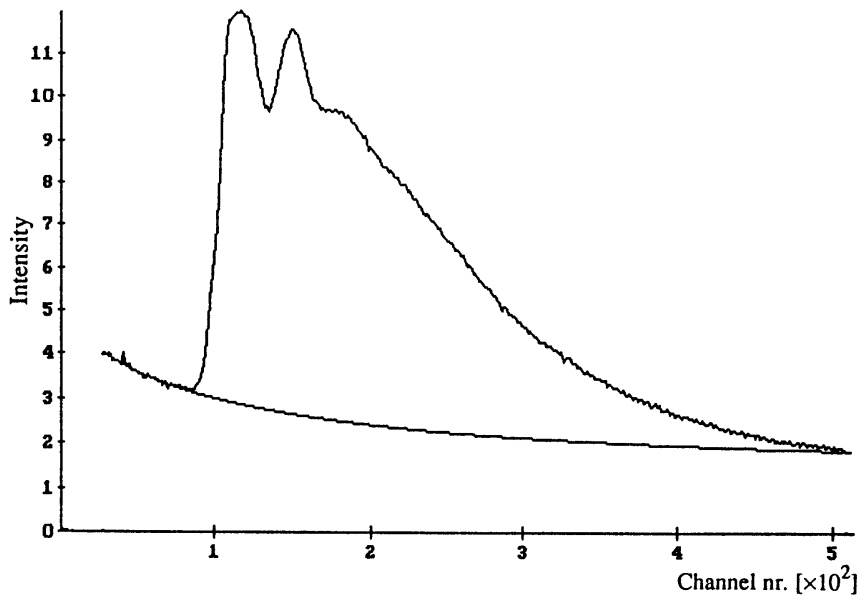

e)

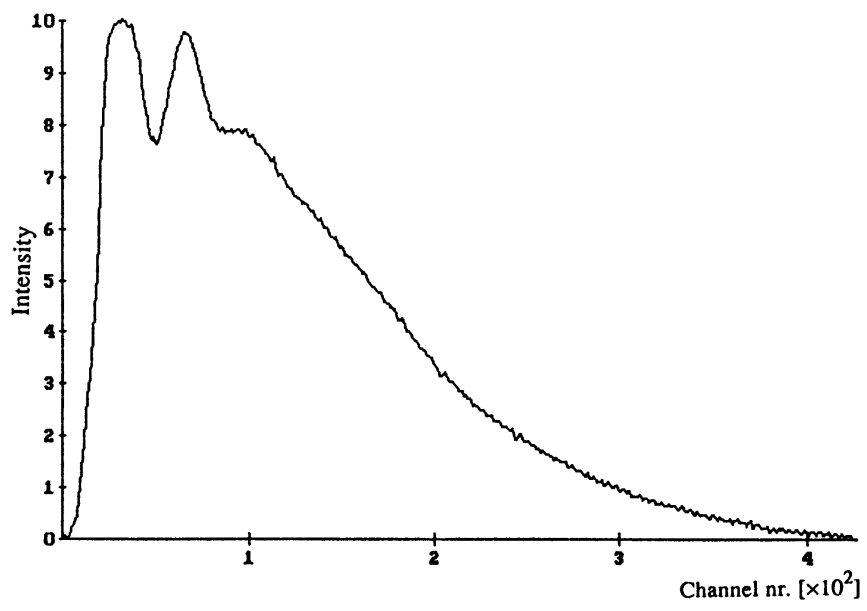

Fig. 1. - (continued) 
An inaccurate choice of this position causes a non-linear deformation of the $k$-axis which may be regarded as a stretching and translation of the interval to adapt to the new $k_{\min }$ and $k_{\max }$ values followed by a non-linear deformation within the interval. This means that a selection of the threshold position to the right (towards higher energies) of its real position will result in a reduction of the measured interatomic distances (and vice versa) with broadened peaks due to the non-linear effects [5].

In the present analysis the inflexion point of the absorption edge has been used as the energy origin. In order to compare the results from this approach to a more correct assumption, this position was also determined from the a priori knowledge about interatomic distances. All three cases (thick spectrum with and without deconvolution and the thin spectrum) consistently determine the "correct" position to about $6 \mathrm{eV}$ below the inflexion point.

ISOLATION OF THE OSCILLATORY COMPONENT. - The oscillatory part, $X(E)$, of the core-loss intensity, $J(E)$, is obtained by normalizing the measured intensity to the intensity corresponding to the single isolated atom case, $A(E)$, i.e. the intensity which would have been measured if neighboring atoms were absent.

$$
X(E)=\frac{J(E)}{A(E)}-1
$$

In general $A(E)$ is not directly available experimentally and can not be theoretically calculated with sufficient accuracy. An empirical approximation can however be obtained by fitting a polynomial function or a continuous combination of polynomials through $J(E)$. The fact that the background should correctly follow the overall trend of the data but not the EXELFS modulations themselves (otherwise false structure will appear in RDF at small values [4]), requires use of low-order polynomials and not too short branches.

With a thick sample without deconvolution, the measured background will, due to plural scattering, deviate considerably from the intensity corresponding to the single isolated atom case, $A(E)$, (see Fig. 2b). This deviation will cause an inaccurate amplification of the modulations. This effect can, however, be partially compensated (during the $k$-dependent amplification prior to the Fourier transform). Furthermore, since the uncompensated part to small degree will affect the width of all peaks in the radial distance diagram equally, its effect on the determination of interatomic distances is often negligible.

An alternative method for isolating the oscillatory part, is by analyzing the second derivative of the spectrum. In this case the derivation must be followed by a sharp low-pass filter to reduce noise. The advantages of this method are that it is easily implemented and that the results are operator independent. A disadvantage is that it contains a high-pass filter reducing the lower frequencies more than the higher frequencies. Another disadvantage is that it is not applicable in all cases, i.e. when the residual background is large. This makes further analysis very difficult (particularly the compensation of the effects of $k$-dependence).

Figures $2 \mathrm{c}$ and $2 \mathrm{~d}$ show the same data as in figure $2 \mathrm{~b}$ i.e. non deconvoluted and deconvoluted data, respectively, with corresponding polynomial backgrounds. These backgrounds consist each of three partitions of a third order polynomial. The result of these background subtractions are shown in figures $2 \mathrm{e}$ and $2 \mathrm{f}$. The inflexion point was, as was mentioned above, used as the origin of the energy-axis.

SELECTION OF THE ANALYSIS INTERVAL - To avoid influence from the near-edge effects and to reduce the sensitivity to deviations in the selection of threshold-energy position, the start point of the analyzing interval should be chosen at about $25 \mathrm{eV}\left(26 \mathrm{~nm}^{-1}\right)$. The end point of the interval should be chosen to maximize the information content, since beyond a certain point increase of 

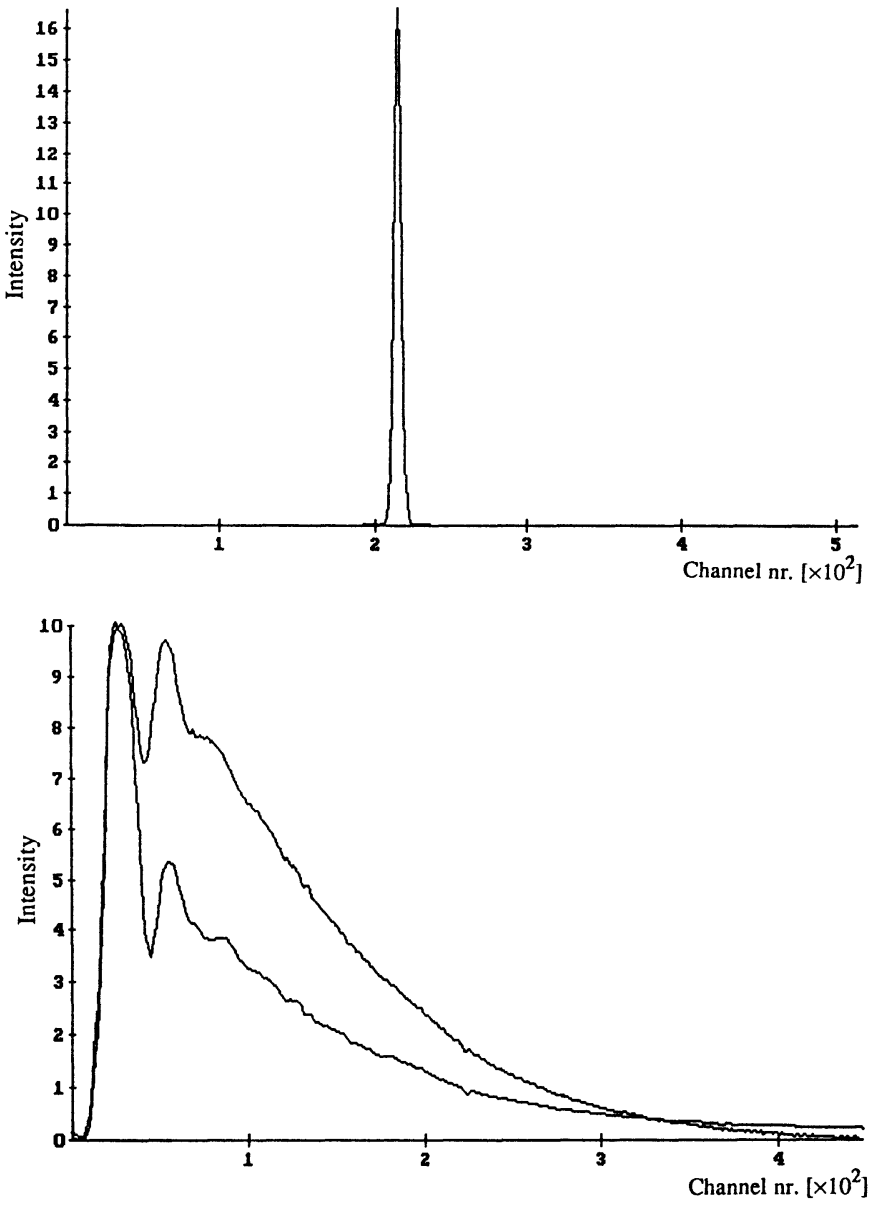

b)

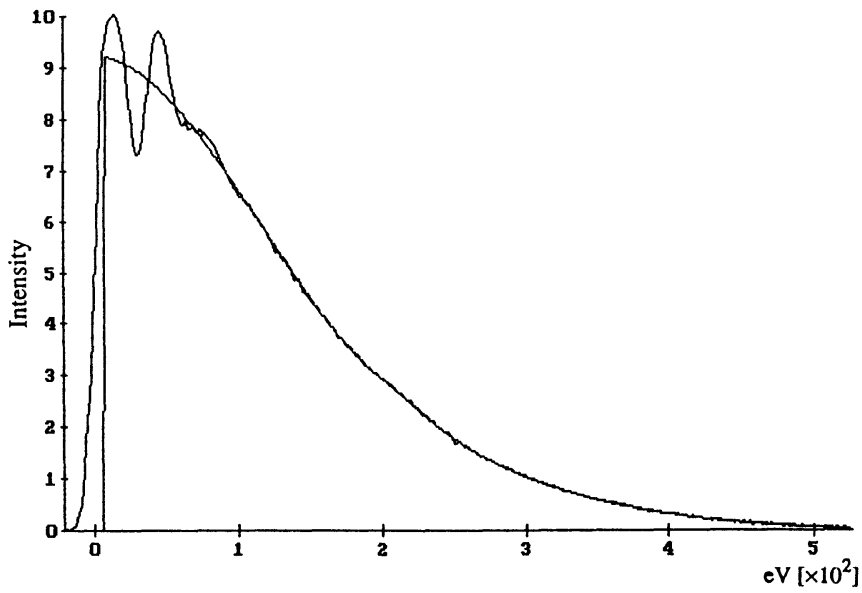

c)

Fig. 2. - (a) a Gaussian function with a width of 5.7 channels. (b) the energy-loss spectrum of the thick sample i.e. figure $1 \mathrm{~b}$, after pre-edge background subtraction, before and after deconvolution. (c) and (d) are non deconvoluted and deconvoluted spectra, respectively, with corresponding backgrounds. (e) and (f) are (c) and (d) after background removal. 


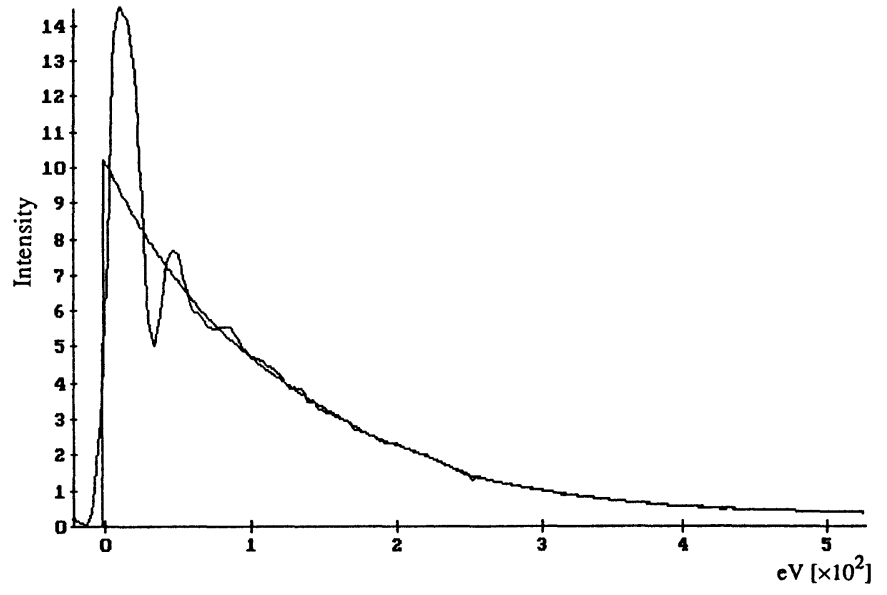

d)

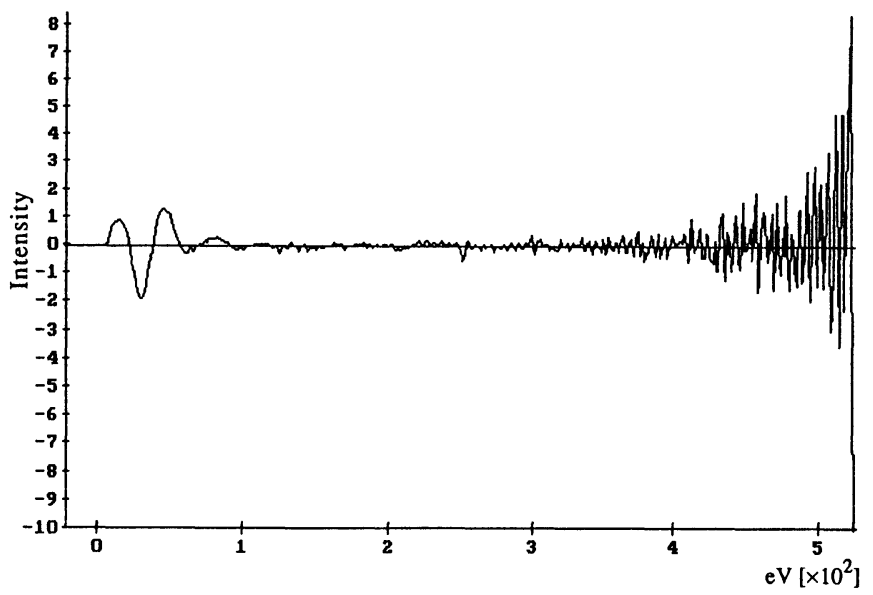

e)

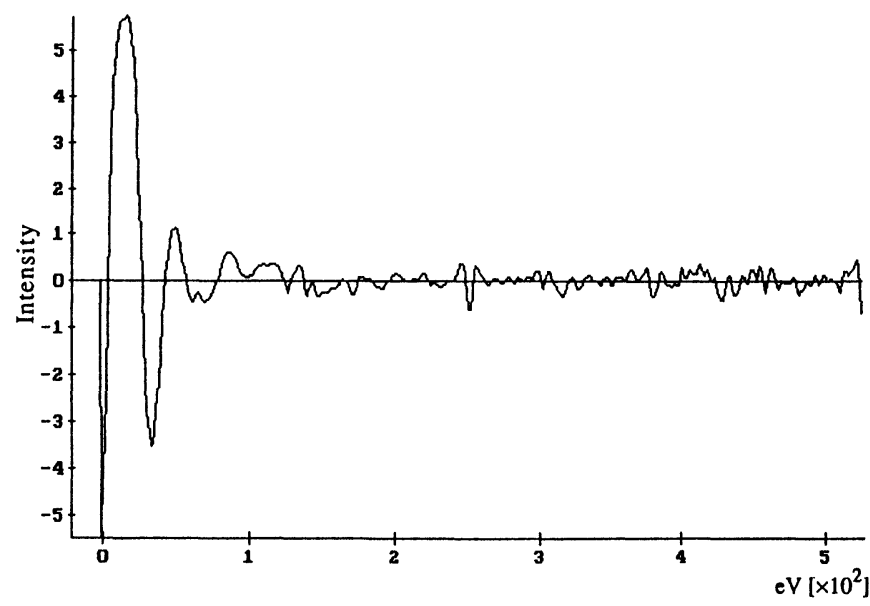

Fig. 2. - (continued) 
the interval will mainly increase the noise. However, the multiplication by a window function (see below) before the Fourier transform introduces strong damping at the beginning and the end of the analyzing interval reducing its effective length. This unintended reduction may be compensated by extending the data interval below and above the prior end points.

The short data interval and the presence of noise introduces errors in the determination of interatomic distances (i.e. of the peak positions in the magnitude spectrum of the Fourier transform). The deviations can vary from zero to a few percent. Therefore, it is often advisable to average results from the analysis with different intervals to improve reliability.

The intervals chosen from the data in figures $2 \mathrm{e}$ and $2 \mathrm{f}$, respectively, are shown in figures $3 \mathrm{a}$ and $3 b$. In both cases the energy interval is from 6 to $520 \mathrm{eV}$ (i.e. 13 to $1171 / \mathrm{nm}$ ).

SCALE CONVERSION. - The further analysis requires resampling of the data so that it becomes equally spaced in electron wave-number space, i.e. a scale conversion from $X_{(E)}$ to $X_{(k)}$ followed by some interpolation in the $k$-space must be performed. Studies of the simulated data have shown that a simple linear interpolation is sufficient.

After this step the interference amplitudes can be regarded as "pure" EXELFS modulations, i.e. they can be described by the equation (1). Figures $3 \mathrm{c}$ and $3 \mathrm{~d}$ show the result of the scale conversion of modulations in figures $3 \mathrm{a}$ and $3 \mathrm{~b}$, respectively.

PREPARING FOR FOURIER TRANSFORM. - Before attempting to Fourier transform the corrected interference amplitude, a compensation for the influence of the $k$-dependent factors (i.e. $1 / k$, $f_{j}(k)$ and the $\sigma$-term, see Eq. (1)) should be performed. This is usually achieved by multiplying with $k^{2}$ or $k^{3}$, where the actual choice depends on measurement parameters such as the noise level. The data should also be multiplied by a window function to minimize the truncation effects after the Fourier transformation. A possible window function varies $\operatorname{asc}^{2} i$ at the ends of the data range but is flat over $50 \%$ of the central region.

Another adjustment made before the Fourier transformation is the addition of a constant level in order to remove the zero frequency component.

The fact that the typical data intervals available for FFT usually are short compared to the wavelengths of the principal modulations [8], implies that the interesting part of the Fourier transform is sparsely sampled. A zero-extention of the data before the Fourier transform will improve the sampling [5]. However, the effect of this operation is to perform an appropriate interpolation between the original sample points.

Figures $4 \mathrm{a}, 4 \mathrm{~b}$ and $4 \mathrm{c}$ show the modulations after multiplying by $k^{2}$ and the window function. $4 \mathrm{a}$ and $4 \mathrm{~b}$ are due to the modulations in figures $3 \mathrm{c}$ and $3 \mathrm{~d}$, respectively (non deconvoluted and deconvoluted data). $4 a$ is obtained from the spectrum in figure 1f, i.e. the thin spectrum.

FOURIER TRANSFORM. - The inter-atomic distances are obtained after a Fourier transform of the interference data followed by a correction for the phase shift effect [3].

In practice, a discrete Fourier transform is used, and the (non phase shift corrected) interatomic distances are deduced directly from the positions of the peaks in the magnitude spectrum. The width of the FFT peaks are mainly caused by the short $k$-range [9], but are also affected by an insufficient compensation for the $k$-dependence, by the window function, and by errors in the position of the threshold-energy. To the extent that the inelastic mean free path, $\lambda_{i}$, can be considered to be independent of $k$, the relative intensities of the peaks are due to the following relationship:

$$
X(k) \propto \sum_{j} \frac{N_{j}}{r_{j}^{2}} \cdot \mathrm{e}^{-2 \cdot \frac{r_{j}}{\lambda_{i}}}
$$



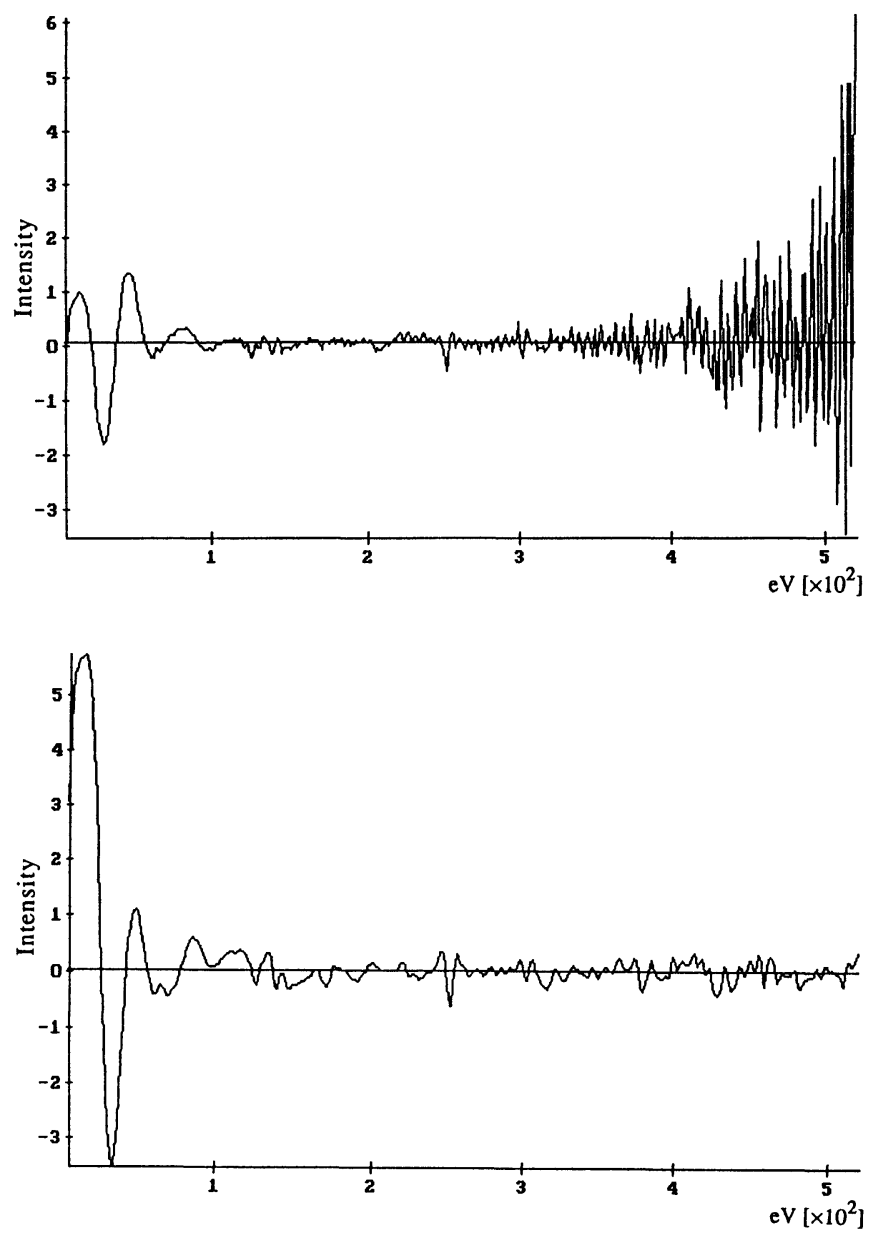

b)

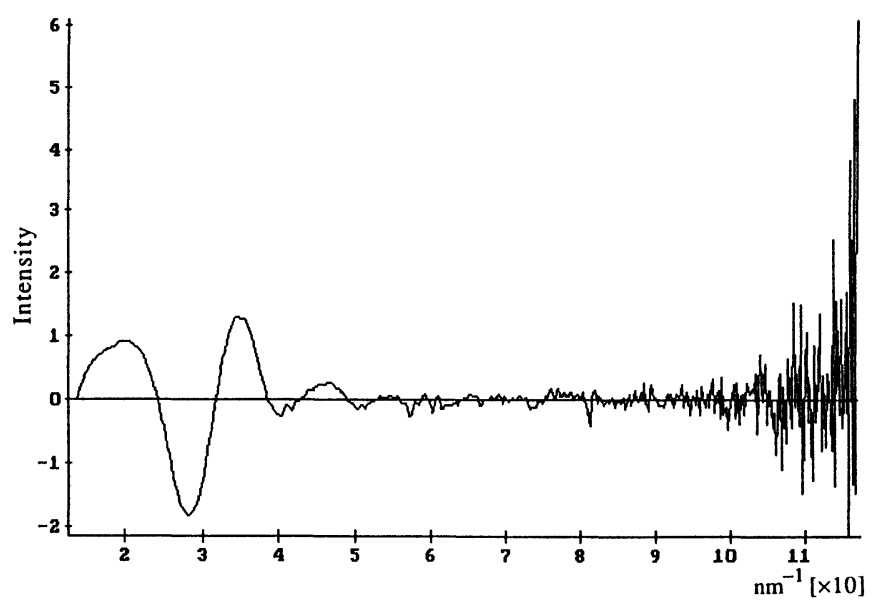

c)

Fig. 3. - (a) and (b) are the spectra in figure $2 \mathrm{e}$ respectively $2 \mathrm{f}$ after selection of the analyzing interval. (c) and (d) are (a) respectively (b) after scale conversion from energy to $k$-space. 


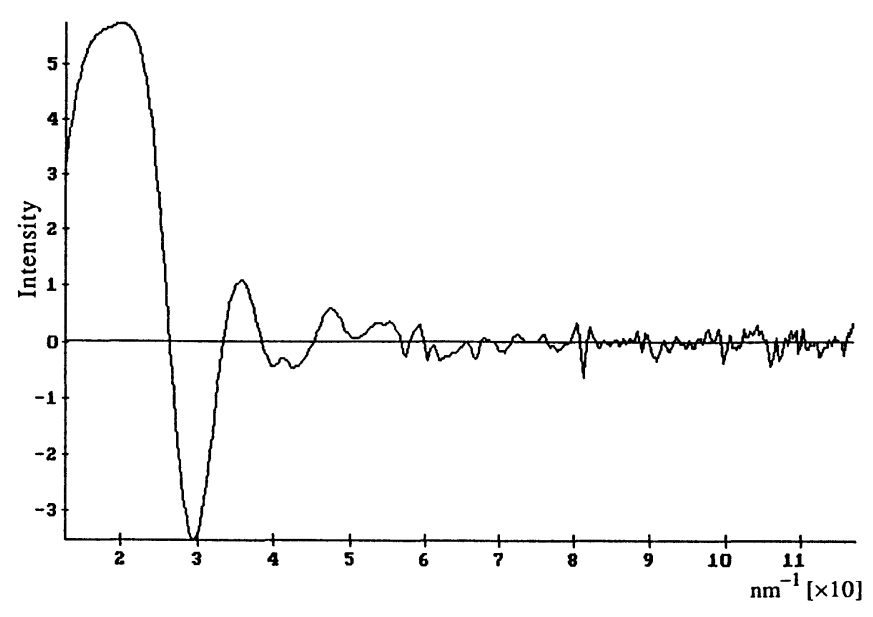

Fig. 3. - (continued)

The phase shift due to the peaks is the phase of the corresponding frequency at the beginning of the $k$-interval $\left(k_{\min }\right)$. This means that, in theory at least, extending the wave from $k_{\min }$ to the $k$-edge position should lead to an estimation of the $\phi_{0}$, with the assumption that phase shift can be written as:

$$
\phi_{j}(k)=\phi_{0}+\phi_{1}(k)
$$

$\phi_{0}$ is very sensitive to the deviations in the peak positions and hence, in order to be able to determine the correct value of $\phi_{0}$, the position of the peaks must be very accurately determined.

Figures $5 \mathrm{a}, 5 \mathrm{~b}$ and $5 \mathrm{c}$ show the magnitudes of the Fourier transforms of the figures $4 \mathrm{a}, 4 \mathrm{~b}$ and $4 \mathrm{c}$, respectively (i.e. non deconvoluted, deconvoluted and thin data), before multiplication by $k^{2}$. In all three cases, the area above $4 \AA$ contains either noise induced, or strongly noise contaminated peaks (due to the low intensity of the high frequency peaks). This means that the interpretation of peaks in this region should be avoided.

Figures $6 a, 6 b$ and $6 c$ show the magnitude of the Fourier transforms of figures $4 a, 4 b$ and $4 c$, respectively (i.e. non deconvoluted, deconvoluted and thin spectrum). Figure $6 \mathrm{~d}$ is same as $6 \mathrm{~b}$ but with "correct" threshold-energy position.

The magnitude of the Fourier transform of the thick sample without deconvolution (Fig. 6a) has a peak at $0.52 \AA$ which can either be a result of insufficient background subtraction or plural scattering, or both. Other peak positions below $4 \AA$ are $0.96,1.75,2.25,2.82$ and $3.68 \AA$. A comparison with the non phase corrected interatomic distances in graphite measured using other techniques, i.e. $1.12,2.16,2.54,3.05$ and $3.47 \AA$, gives the respective deviations: $+14.3 \%$, $+19 \%$, $+11.4 \%,+7.5 \%$ and $-6.4 \%$.

A strong deviation in the last peak position is expected, due to the low intensity of this peak and the high noise level (see Fig. 5a). It must also be noted that the phase shift corrections are obtained by taking the average slope $(0.3 \AA)$ of the phase shift for C-C calculated by Teo and Lee [3].

In the case of deconvoluted data (i.e. Fig. $6 \mathrm{~b}$ ) the peak positions below $4 \AA$ are $1.07,1.98,2.52$, 3.05 and $3.61 \AA$, which yield $+4.5 \%,+8.3 \%,+0.8 \%, 0.0 \%$, and $-4.3 \%$ deviations, respectively, from the known values. Even in this case a low frequency component near the first peak is visible, which can be explained as being due to insufficient background subtraction. Again, due to the low 


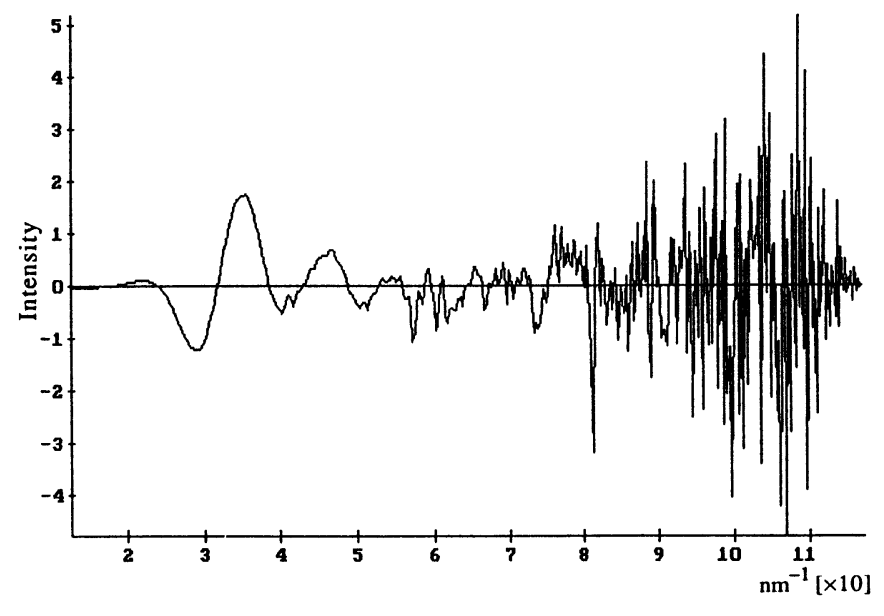

a)

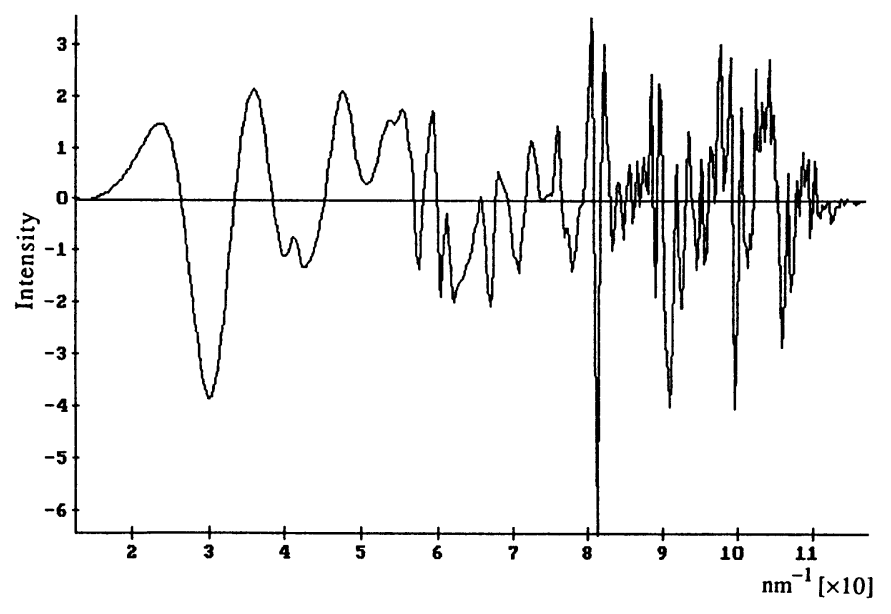

b)

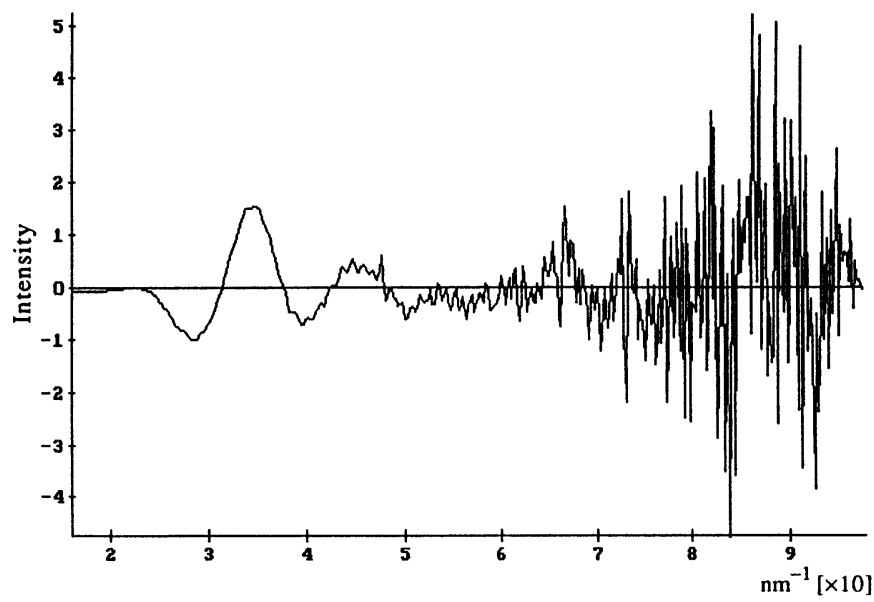

c)

Fig. 4. - (a) and (b) are the spectra from figures $3 \mathrm{c}$ and $3 \mathrm{~d}$ i.e. non deconvoluted and deconvoluted spectrums, respectively, after multiplying by $k^{2}$ and the window function. (c) is the corresponding spectrum from 1f, i.e. the thin spectrum. 


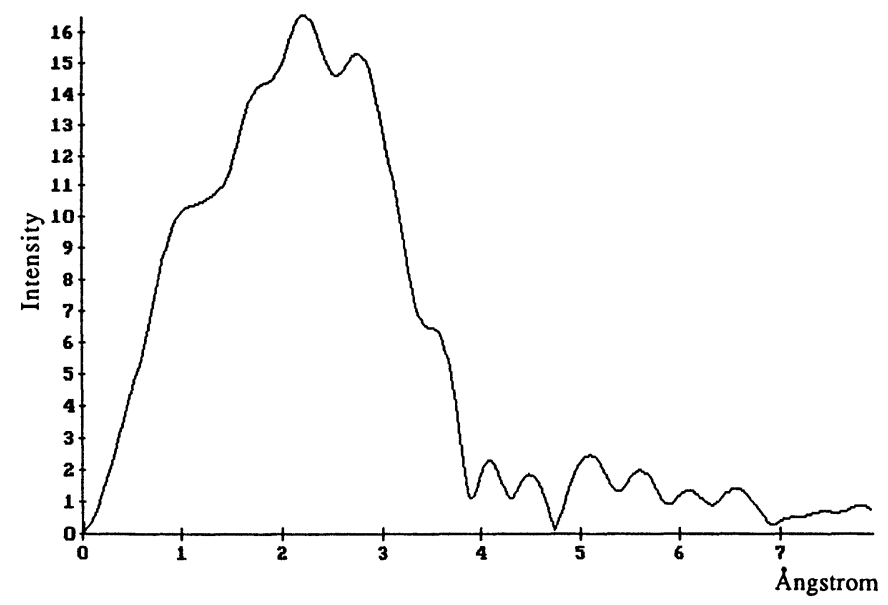

a)

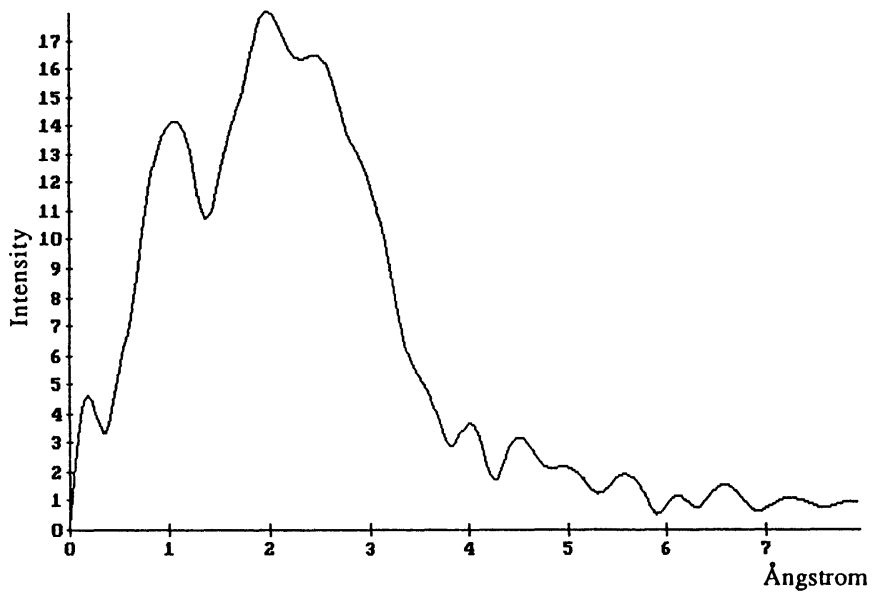

b)

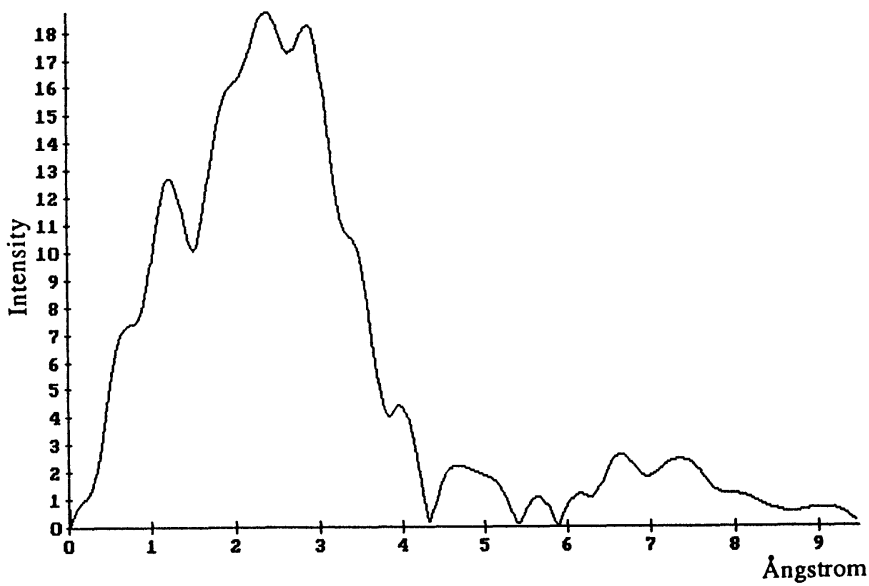

c)

Fig. 5. - (a), (b) and (c) are the magnitude of the Fourier transform, corresponding to the modulations in figures $4 \mathrm{a}, 4 \mathrm{~b}$ and $4 \mathrm{c}$ i.e. non deconvoluted, deconvoluted and thin spectrum, respectively, before multiplication by the $k^{2}$. Observe that the peaks above $4 \AA$ are either due to noise or are strongly contaminated by it. 


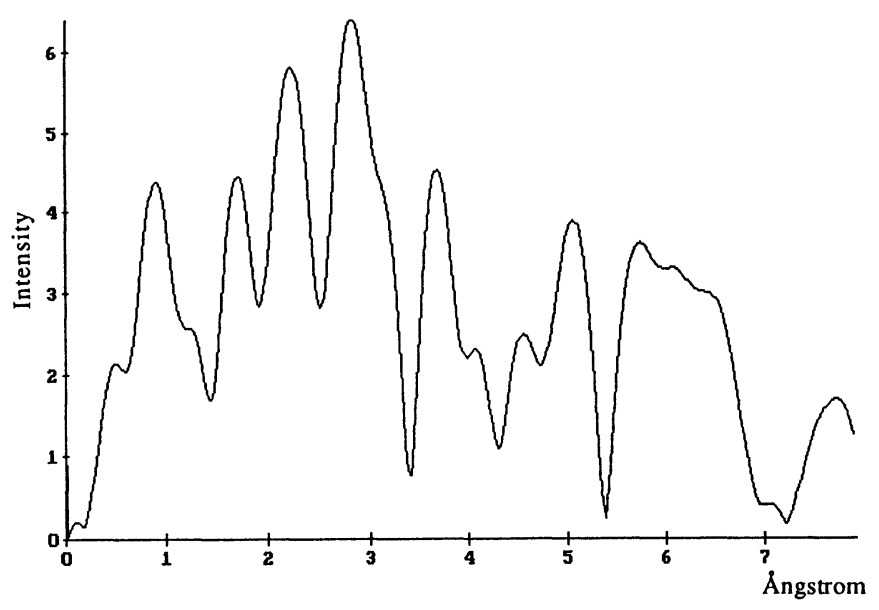

a)

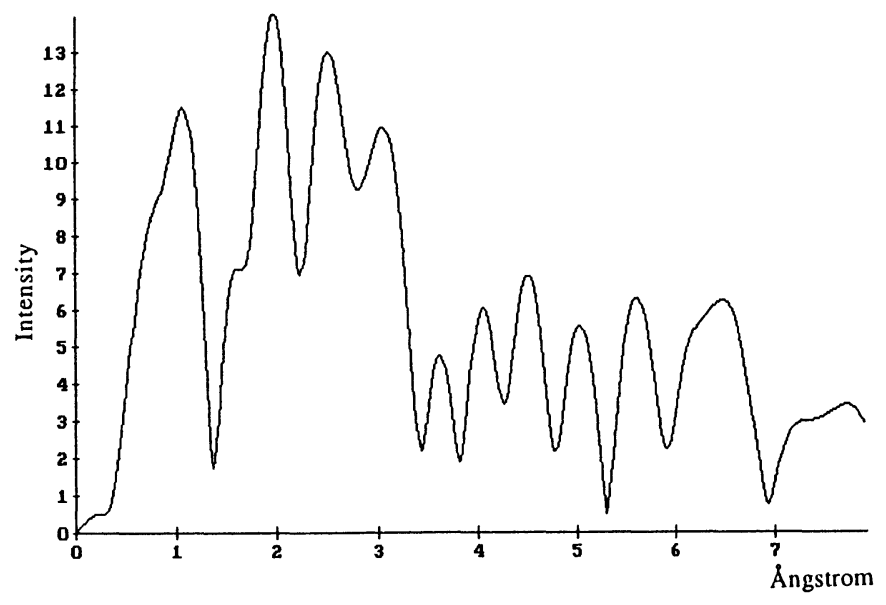

b)

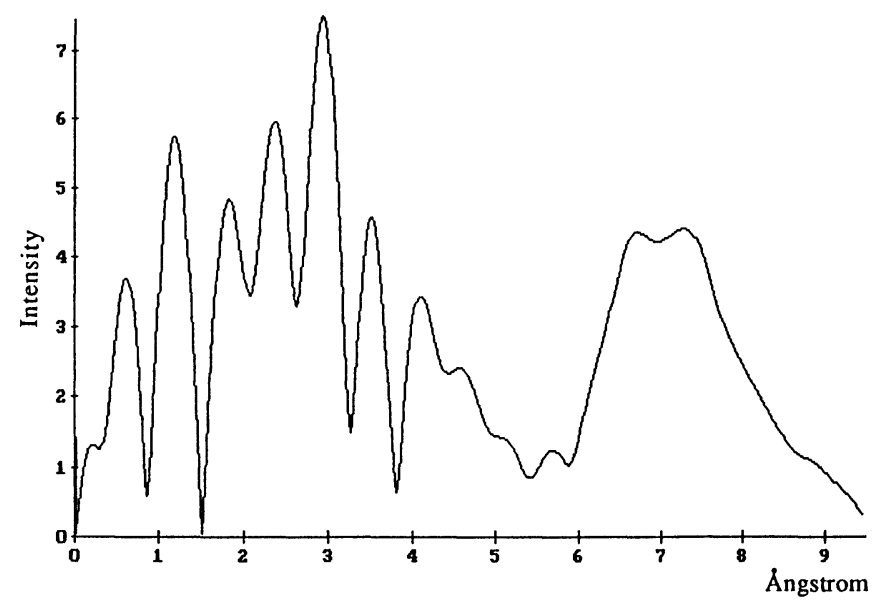

c)

Fig. 6. - (a), (b) and (c) are the magnitude of the Fourier transform of the modulations in figures 4a, $4 \mathrm{~b}$ and $4 \mathrm{c}$ i.e. the non deconvoluted, deconvoluted and the thin spectrum, respectively. (d) is same as (b) but with correct threshold-energy position determined by a priori knowledge about the interatomic distances. 


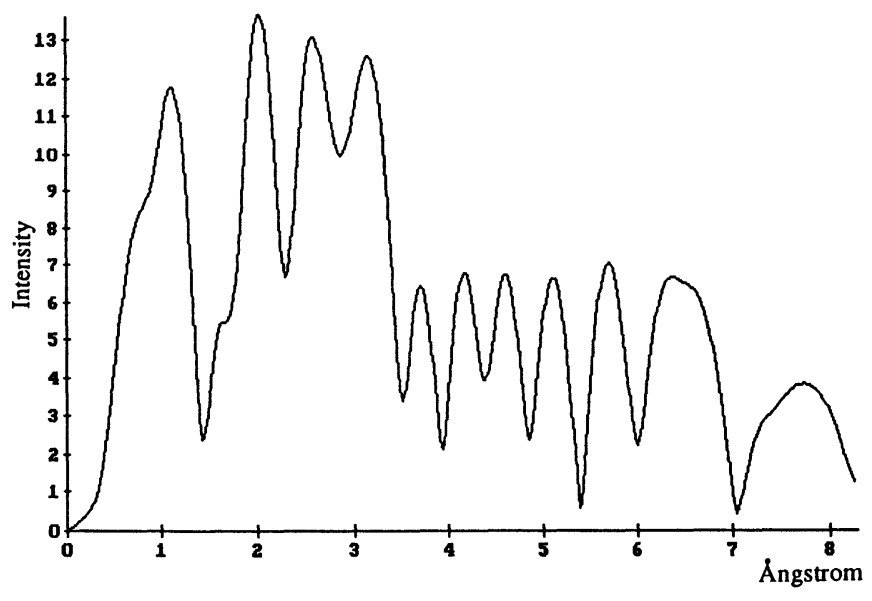

d)

Fig. 6. - (continued)

signal to noise ratio a strong deviation in the position of the last peak was expected.

The position of the peaks between 1 and $4 \AA$ in the case of the thin spectrum (Fig. $6 \mathrm{c}$ ) are 1.20, $1.83,2.39,2.96$ and $3.53 \AA$, which means $-7.1 \%,+15.3 \%,+5.9 \%,+3.0 \%$ and $-2.0 \%$ deviations, respectively, from the known values.

The average of the absolute deviations of the first four peak positions in the case of the thick sample without deconvolution is $13.0 \%$. The same value after deconvolution is $3.4 \%$ while the value for the thin spectrum is $7.8 \%$. Using the "correct" threshold-energy position these values become $10.6 \%, 2.8 \%$ and $6.6 \%$ respectively. That is, the use of the "correct" position caused an improvement proportional to the previous deviations.

\section{Concluding remarks.}

The deconvolution technique based on the Fourier transform and convolution with a Gaussian function has successfully been used to remove the plural scattering from the inner-shell edges. This removal has drastically reduced systematic errors in the EXELFS analysis due to sample thickness. In the case of non deconvoluted data the first four non phase shift corrected interatomic distances has an average of $\mathbf{1 3 . 0 \%}$ deviation from the known values since the same average for the deconvoluted data is $3.4 \%$. An analysis without deconvolution of the relatively thin graphite sample gave the deviation 7.8\%. Part of the discrepancy between these two cases is due to plural scattering and part is due to the fact that the improved signal to background ratio after removal of effects of the plural scattering increases the accuracy and precision in the further analysis after deconvolution.

The actual choice of analysis interval influences the result. To decrease this inaccuracy, the non phase shift corrected interatomic distances can be derived as the mean of the results from a few different intervals.

A reliable method for taking care of the pre-edge background is to fit a monotonically decreasing smooth function, which asymptotically approaches a constant background level, to data in the 
pre-edge region. The oscillatory part of the core-loss intensity can be obtained by empirically fitting a third order polynomial function through the whole or different partitions of the core-loss intensity.

\section{References}

[1] KinCaide B.M., A.E. and PLATZMAN P.M., Carbon K-edge in graphite measured using electron energyloss spectroscopy, Phys. Rev. Lett. 40 (1978) 1296-1299.

[2] STERN E.A., Theory of the extended X-ray absorption fine structure. Phys. Rev. B 10 (1974) 3027-3037.

[3] TeO B.-K. and LeE P.A., $A b$ initio calculations of amplitude and phase functions for extended X-ray absorption fine structure spectroscopy. J. Am. Chem. Soc. 101 (1979) 2815-2832.

[4] EGERTON R.F., Electron Energy-Loss Spectroscopy in the Electron Microscope pp. 279-281.

[5] TAFreshi M.A., BOHM Ch. and CSILlAG S., Microsc. Microanal. Microstruct. 1 (1990) 199-213.

[6] EGERTON R.F. and CROZIER P.A., The use of Fourier techniques in electron energy-loss spectroscopy, Scanning Micros. Suppl. 2. (1988) pp. 245-254.

[7] BAXTER D.V., The Selection of $E_{0}$ for EXELFS Spectra of Disordered Systems. EXAFS and Near Edge Structure III, K.O. Hodgsen, B. Hedman and J.E. Penner-Hahn Eds. (Springer-Verlag) pp. 77-79.

[8] BouRdillon A.J., El MARShRI S.M. and ForTY A.J., Application of extended electron energy loss fine structure to the study of aluminium oxide films, Philos. Mag. 49 (1984) 341-352.

[9] DiSKO M.M., KRIVANEK O.L. and REZ P., Orientation dependent extended fine structure in electron energy-loss spectra, Phys. Rev. B 25 (1982) 4252-4255. 